\title{
Icariin Promotes the Osteogenesis of Bone Marrow Mesenchymal Stem Cells through Regulating Sclerostin and Activating the Wnt/ $\beta$-Catenin Signaling Pathway
}

\author{
Jianliang Gao, Shouyu Xiang, Xiao Wei, Ram Ishwar Yadav, Menghu Han, Weihao Zheng, \\ Lili Zhao, Yichuan Shi, and Yanming Cao iD
}

Department of Orthopedics, The Second Affiliated Hospital of Guangzhou Medical University, Guangzhou, China

Correspondence should be addressed to Yanming Cao; 13729813888@126.com

Received 4 December 2020; Revised 21 December 2020; Accepted 2 January 2021; Published 23 January 2021

Academic Editor: Chaohong Li

Copyright (C) 2021 Jianliang Gao et al. This is an open access article distributed under the Creative Commons Attribution License, which permits unrestricted use, distribution, and reproduction in any medium, provided the original work is properly cited.

\begin{abstract}
Osteoporosis (OP) is a metabolic disease characterized by decreased bone mass and increased risk of fragility fractures, which significantly reduces the quality of life. Stem cell-based therapies, especially using bone marrow mesenchymal stem cells (BMSCs), are a promising strategy for treating OP. Nevertheless, the survival and differentiation rates of the transplanted BMSCs are low, which limits their therapeutic efficiency. Icariin (ICA) is a traditional Chinese medicine formulation that is prescribed for tonifying the kidneys. It also promotes the proliferation and osteogenic differentiation of BMSCs, although the specific mechanism remains unclear. Based on our previous research, we hypothesized that ICA promotes bone formation via the sclerostin/Wnt/ $\beta$-catenin signaling pathway. We isolated rat BMSCs and transfected them with sclerostin gene (SOST) overexpressing or knockdown constructs and assessed osteogenic induction in the presence or absence of ICA. Sclerostin significantly inhibited BMSC proliferation and osteogenic differentiation, whereas the presence of ICA not only increased the number of viable BMSCs but also enhanced ALP activity and formation of calcium nodules during osteogenic induction. In addition, the osteogenic genes including Runx2, $\beta$-catenin, and c-myc as well as antioxidant factors (Prdx1, Cata, and Nqo1) were downregulated by sclerostin and restored by ICA treatment. Mechanistically, ICA exerted these effects by activating the $\mathrm{Wnt} / \beta$-catenin pathway. In conclusion, ICA can promote the proliferation and osteogenic differentiation of BMSCs in situ and therefore may enhance the therapeutic efficiency of BMSC transplantation in OP.
\end{abstract}

\section{Introduction}

Osteoporosis (OP) is a common skeletal disease characterized by reduced bone mineral density (BMD), bone microstructure deterioration, and an increased risk of fragility fractures $[1,2]$. The incidence of OP has risen sharply with the aging of the global population, and currently, one-third of women and one-fifth of men are afflicted worldwide [3]. The antiosteoporosis drugs approved at present are bone resorption inhibitors [4], and their long-term intake can increase the risk of jaw osteonecrosis and atypical femoral fractures, thereby limiting their clinical application [5]. Therefore, it is necessary to develop novel treatment strategies for OP.
Recent studies show that bone marrow mesenchymal stem cells (BMSCs) can repair bone defects in several animal models [6] and are a promising tool for bone regeneration in OP [7]. However, the survival and differentiation rates of the transplanted BMSCs are low, which significantly reduces the efficacy of BMSC-based regenerative therapy [8]. Therefore, enhancing the proliferation and osteogenesis differentiation of BMSCs in situ can significantly improve therapeutic efficacy. Sclerostin is an osteoinhibitory protein encoded by the SOST gene that is secreted by bone cells [9] and inhibits osteoblast activity by inactivating the $\mathrm{Wnt} / \beta$-catenin pathway [10]. The SOST-/- mice show accelerated osteoblast differentiation via $\beta$-catenin activation [11], which suggests that knocking down the 


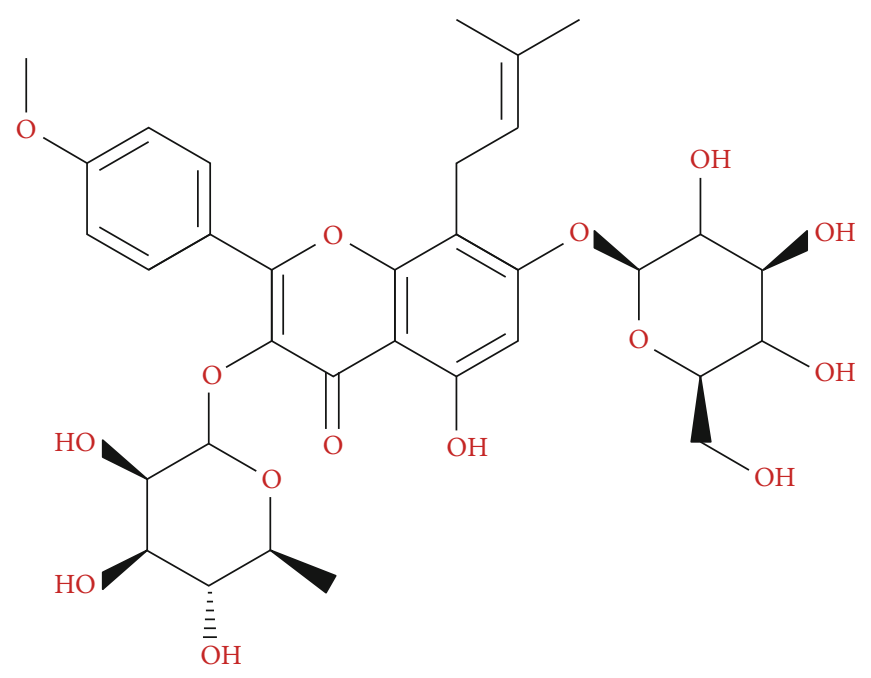

FIgUre 1: The chemical structure of ICA.

SOST gene may enhance osteogenic differentiation of BMSCs and promote bone renewal.

Icariin (ICA, chemical structure shown in Figure 1) is the main active flavonoid glycoside in the Chinese herbal medicine Herba Epimedii, which is used to treat renal disorders and prevent bone loss [12]. Recent studies show that ICA can effectively treat postmenopausal $\mathrm{OP}$ and promote bone formation [13] by stimulating BMSC proliferation and osteogenic differentiation [14]. In addition, the Chinese medicinal formulation Zuogui pills can also regulate BMSC osteogenic differentiation via the $\mathrm{Wnt} / \beta$-catenin signaling pathway [15]. The aim of this study was to elucidate the mechanistic basis of the proosteogenic function of ICA, with specific focus on the sclerostin-Wnt/ $\beta$-catenin signaling pathway. To this end, we isolated rat BMSCs and transfected them with SOST overexpression or knockdown constructs and treated them with ICA during osteogenic induction.

\section{Materials and Methods}

2.1. Animals and Ethics. Twelve-week-old female SpragueDawley (SD) rats weighing 200-300 g were purchased from the Experimental Animal Center of Sun Yat-sen University (Guangzhou, China, Certificate SCXK (Guangdong) 20160029). Animal experiments were performed in accordance with the guidelines of the Regulations of the People's Republic of China on the Administration of Experimental Animals and approved by the Animal Ethics Committee of the Second Hospital Affiliated with Guangzhou Medical University.

2.2. Chemical Reagents and Drugs. Dulbecco minimum essential medium (DMEM), fetal bovine serum (FBS), potassium phosphate buffer saline (PBS), and penicillinstreptomycin were purchased from Hyclone (Logan, Utah, USA). Icariin (ICA, purity $>94 \%$ ) and dimethyl sulfoxide (DMSO) were obtained from Sigma (Steinheim, Germany). The alkaline phosphatase (ALP) staining kit (BCIP/NBT kit) was obtained from Beyotime Biotechnology (Shanghai, China) and the ALP activity measurement kit from Nanjing Jiancheng Biotech (Nanjing, China). Anti- $\beta$-catenin anti- body was purchased from GeneTex (Southern California, USA) and the antibodies targeting GSK- $\beta$ and p-GSK- $\beta$ from GST (Hangzhou, China). Secondary antibodies were obtained from Abcam (Shanghai, China).

2.3. BMSC Extraction and Culture. The animals were euthanized by cervical dislocation, and the tibia and femurs were removed. The bone marrow cavities were flushed repeatedly with $10 \%$ FBS-supplemented DMEM using a sterile $10 \mathrm{ml}$ syringe to prepare a single cell suspension. The cells were seeded into a $25 \mathrm{~cm}^{2}$ flask and cultured at $37^{\circ} \mathrm{C}$ under $5 \%$ $\mathrm{CO}_{2}$ [16]. The medium was changed every 3 days, and the cells were observed regularly under an inverted microscope. After culturing for 7-10 days, the 80\%-90\% confluent monolayer was harvested with $0.25 \%$ EDTA-trypsin, washed at $1000 \mathrm{rpm}$ for 5 minutes, and resuspended in complete DMEM for subculturing at $1: 2$ ratio.

2.4. Flow Cytometry. BMSCs $\left(1 \times 10^{6}\right.$ cells $\left./ \mathrm{ml}\right)$ were incubated with phycoerythrin-labeled anti-CD90, anti-CD44, anti-CD31, or anti-CD34 antibodies at room temperature in the dark. After washing twice with PBS at $800 \mathrm{rpm}$ for 5 minutes, the cells were acquired by flow cytometry [17].

2.5. Vector Construction and Lentivirus Production. The SOST overexpression and shRNA lentiviruses were constructed by Suzhou Genewiz. The SOST gene was amplified using the following primers: SOST forward ATGCAGCTC TCACTAGCCCCTTGCC and SOST reverse CTAGTA GGCGTTCTCCAGCTCCGCCTGG. The SOST shRNA sequences were as follows: forward GGCCTCCTCAGGAA CTAGAGAATTCAAGAGATTCTCTAGTTCCTGAGGA GGCTTTTT and reverse AAAAAGCCTCCTCAGGAA CTAGAGAATCTCTTGAATTCTCTAGTTCCTGAGGAG GCC. The sequences were cloned into L303-CMV.Gene.EF1.GFP-T2A-Puro and L202-CMV.CopGFP.2A.Puro $\mathrm{H} 1$ plasmids, which were transiently transfected into $293 \mathrm{~T}$ packaging cells (Invitrogen, Thermo Fisher Scientific Inc.). The serum-free medium was replaced after 8 hours with complete medium. The cells were cultured for 48 hours, 
TABLe 1: Primers used for target amplification in this study.

\begin{tabular}{|c|c|c|c|}
\hline Name & Accession number & Primer & Sequence $\left(5^{\prime}-3^{\prime}\right)$ \\
\hline Runx2 & NM_053470.1 & $\begin{array}{l}\text { Forward } \\
\text { Reverse }\end{array}$ & $\begin{array}{l}\text { GATGCCTTAGTGCCCAAATGT } \\
\text { GGCTGAAGGGTGAAGAAAGC }\end{array}$ \\
\hline$\beta$-catenin & NM_053357.2 & $\begin{array}{l}\text { Forward } \\
\text { Reverse }\end{array}$ & $\begin{array}{l}\text { TGAGAAACTTGTCCGATGCA } \\
\text { CACTTGGCACACCATCATCT }\end{array}$ \\
\hline$c-m y c$ & NM_012603.2 & $\begin{array}{l}\text { Forward } \\
\text { Reverse }\end{array}$ & $\begin{array}{l}\text { TGTAGTAATTCCAGCGAGAG } \\
\text { CGCAGATTGTAAGTTCCAG }\end{array}$ \\
\hline Prdx1 & NM_057114.1 & $\begin{array}{l}\text { Forward } \\
\text { Reverse }\end{array}$ & $\begin{array}{l}\text { GGATTGGGACCCATGAACAT } \\
\text { GAACTGGAAGGCCTGGACTA }\end{array}$ \\
\hline Cata & NM_012520.2 & $\begin{array}{l}\text { Forward } \\
\text { Reverse }\end{array}$ & $\begin{array}{l}\text { CCATCGCCAGTGGCAATTAC } \\
\text { AGTCCTTGTGAGGCCAAACC }\end{array}$ \\
\hline Nqo1 & NM_017000.3 & $\begin{array}{l}\text { Forward } \\
\text { Reverse }\end{array}$ & $\begin{array}{l}\text { TGTGGCTTCCAGGTCTTAGA } \\
\text { TGACTCCTCCCAGACAGTCT }\end{array}$ \\
\hline GAPDH & NM_017008.3 & $\begin{array}{c}\text { Forward } \\
\text { Reverse }\end{array}$ & $\begin{array}{l}\text { CCCATTCTTCCACCTTTGAT } \\
\text { CAACTGAGGGCCTCTCTCTT }\end{array}$ \\
\hline
\end{tabular}

and the supernatant was collected and concentrated. The virus titer was measured, and the transduction rate was measured by PCR [18].

2.6. BMSC Transfection and Osteogenic Induction. The BMSCs were transduced with the SOST overexpression or shRNA lentiviruses and cultured in osteogenic induction medium with or without $0.1 \mu \mathrm{M}$ ICA. Nontransfected and normal controls were also included. After predetermined time points, osteogenic differentiation was assessed by specific tests.

2.7. CCK-8 Assay. BMSCs were seeded into 96-well plates at the density of $1 \times 10^{4}$ per well, and $10 \mu \mathrm{lCCK}-8$ reagent (Keki Bio, Cat. No. KGA317) was added to each well after 24, 48, and $72 \mathrm{~h}$ of culture. After incubating for 2 hours, the absorbance was measured at $450 \mathrm{~nm}$ using a microplate reader (Thermo Fisher Scientific, Vantaa, Finland).

2.8. Alkaline Phosphatase (ALP) Staining and Activity Assay. The suitable cells were fixed with $10 \%$ formaldehyde for 15 minutes, rinsed with distilled water for 30 seconds, and stained with NBT-BCIP solution (Beyotime Biotechnology, China) at $37^{\circ} \mathrm{C}$ in the dark for 15 minutes [19]. After washing with distilled water for 30 seconds, the stained cells were viewed under a microscope. For the ALP activity assay, the cells were digested with $0.25 \%$ pancrease at room temperature for 2 to 3 minutes and centrifuged. After washing once with PBS, the lysate was analyzed using the ALP assay kit according to the manufacturer's instructions.

2.9. Alizarin Red Staining. The differentiated BMSCs were transferred to a six-well plate and fixed with $2 \mathrm{ml} \mathrm{4 \%} \mathrm{neutral}$ formaldehyde solution for $30 \mathrm{~min}$. After rinsing twice with PBS, $1 \mathrm{ml}$ Alizarin red solution was added to each well, and the cells were stained for 3-5 min. The cells were rinsed 2-3 times and observed under a microscope.

2.10. $R T-q P C R$. Total RNA was extracted using Trizol reagent (Invitrogen, USA) and quantified using a Thermo Scientific Microplate Reader (Thermo, USA). RT-qPCR was conducted using SYBR Green qPCR SuperMix (Invitrogen, California, USA) on the ABI PRISM ${ }^{\circledR} 7500$ Sequence Detection System (Applied Biosystems; Thermo Fisher Scientific, Inc.). The primer sequences are listed in Table 1.

2.11. Western Blotting. Western blotting was performed according to standard protocols [20]. Briefly, the proteins were loaded onto $10 \%$ SDS-PA gels, electrophoresed, and transferred onto polyvinylidene difluoride membranes (Millipore, USA). The blots were probed overnight with anti- $\beta$-catenin, anti-GSK-3 $\beta$, and anti-p-GSK-3 $\beta$ primary antibodies at $4^{\circ} \mathrm{C}$, followed by secondary antibody $(1: 2000$ Southern Biotech) at room temperature for 1 hour. GAPDH ( $1: 1000$, Aksomics, China) was used as the internal control [21]. The positive bands were visualized using an Enhanced Chemiluminescence Western Blot Substrate Kit (Keygen, Nanjing) and analyzed using ImageJ software (National Institutes of Health, Bethesda, MD).

2.12. Statistical Analysis. Data are presented as mean \pm standard deviation (SD). Statistical analyses were performed using SPSS version 16.0 software (SPSS Inc., Chicago, IL, USA). One-way analysis of variance (ANOVA) or Student's $t$-test was used to compare different groups. $P$ values less than 0.05 were considered statistically significant.

\section{Results}

3.1. Characterization of BMSCs. The initial BMSC cultures exhibited adherent cells, colonies, and floating cells (Figure 2(a), A), and the latter disappeared completely by the fifth passage (Figure 2(a), B). Immunophenotypic analysis showed that $99.83 \%$ and $99.78 \%$ of the cells expressed CD90 and CD44, and only $1.15 \%$ and $1.43 \%$ expressed CD31 and CD34, respectively (Figures 2(b)-2(e)), indicating that most cells were BMSCs.

3.2. ICA Promotes Proliferation and Osteogenic Differentiation of BMSCs. As shown in Figure 3, $0.1 \mu \mathrm{M}$ ICA significantly increased proliferation of the BMSCs compared to the untreated controls. Osteogenic differentiation of the BMSCs 


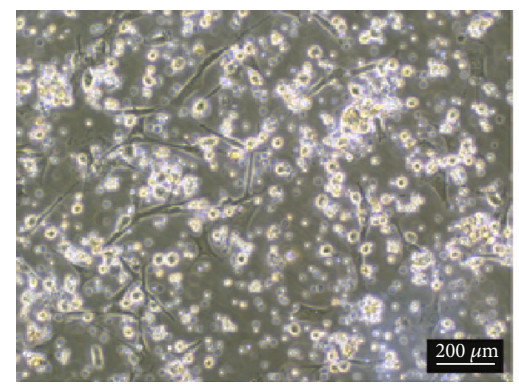

Primary cell of rat BMSCs $\times 100$

(a)

(a)

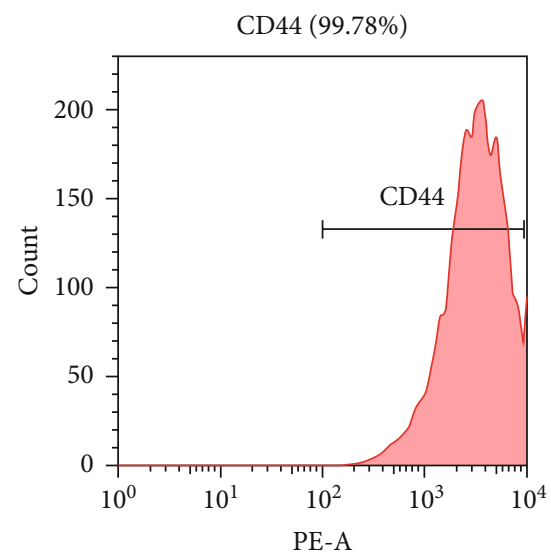

(c)

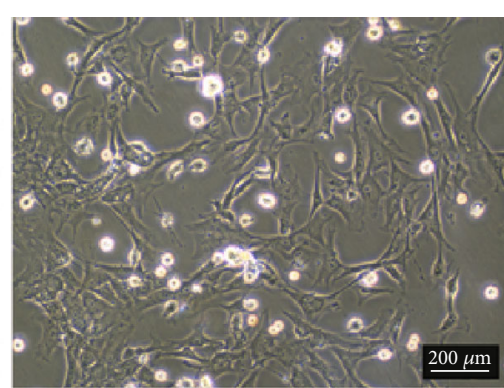

5 passages of rat BMSCs $\times 100$

(b)

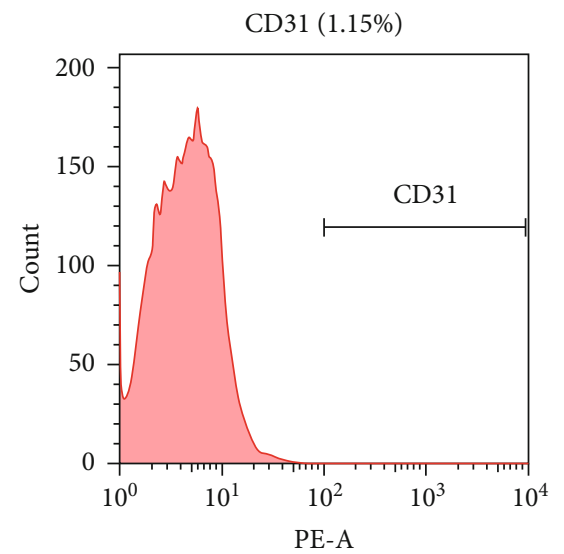

(d)

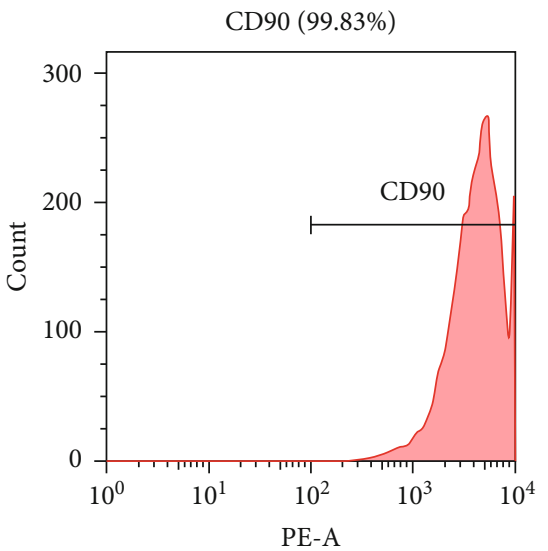

(b)

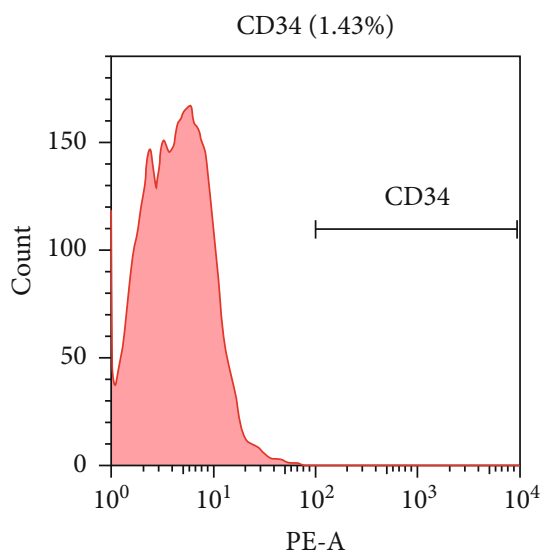

(e)

FIGURE 2: Morphology and phenotypic characterization of BMSCs. (a) Representative images of cultured rBMSCs at the primary passage (A) and passage 5 (B). Flow cytometry plots showing percentage of cells expressing (b) CD90, (c) CD44, (d) CD31, and (e) CD34.

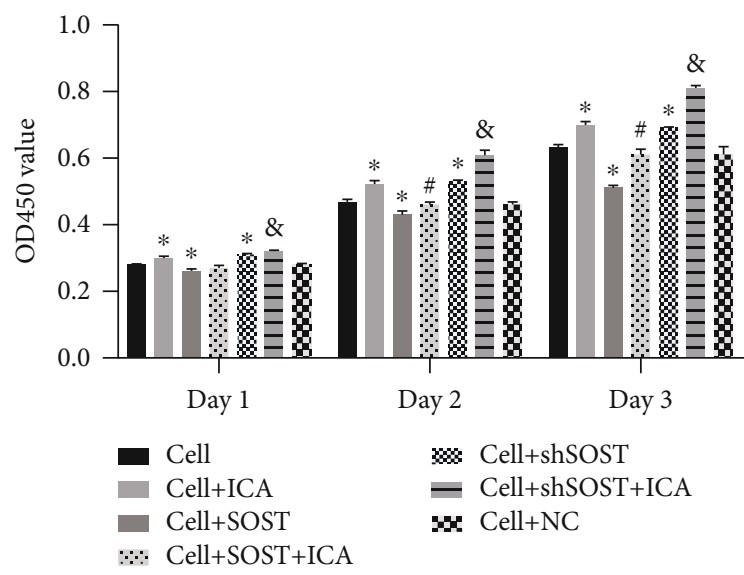

FIGURE 3: Effects of ICA and sclerostin on BMSC proliferation. Percentage of viable cells in the indicated groups at days 1,2 , and 3 postinduction. All data are presented as mean \pm SEM. ${ }^{*} P<0.05$ compared to the control group, ${ }^{\#} P<0.05$ compared to the sclerostin overexpression group, and ${ }^{\&} P<0.05$ compared to the sclerostin knockdown group. was assessed by ALP staining and activity and Alizarin red staining. ICA significantly enhanced ALP levels and activity in the BMSCs compared to the control (Figure 4), which is indicative of early osteoblast differentiation. Furthermore, intense Alizarin red staining in the ICA-treated cells indicated formation of calcified nodules and osteogenic differentiation (Figure 4). Taken together, ICA significantly promoted BMSC proliferation and osteogenic differentiation in vitro.

3.3. ICA Reverses the Inhibitory Effect of Sclerostin on Osteogenic Gene Expression. To ascertain the involvement of sclerostin in ICA-induced osteogenic differentiation of BMSCs, we transfected the cells with SOST overexpression and shRNA constructs prior to ICA treatment. As shown in Figures 3 and 4, SOST overexpression inhibited BMSC proliferation and ALP activity compared to the control group, whereas SOST knockdown had the opposite effect. However, ICA treatment restored proliferative and osteogenic capacity of the SOST-overexpressing BMSCs, which underscores the proosteogenic activity of ICA. Consistent with this, ICA significantly increased the expression levels of osteogenic genes including Runx2, $\beta$-catenin, and c-myc after 4,7 , and 14 days. Similar results were observed in the SOST-knockdown BMSCs as well. In addition, the osteogenic genes were also 
Cell
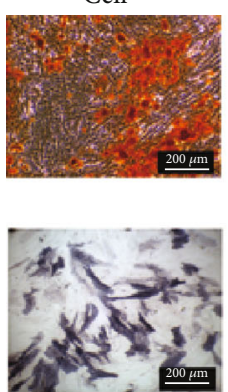

ICA
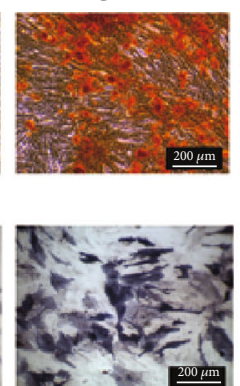

SOST
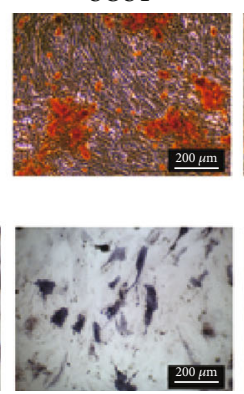

SOST+ICA

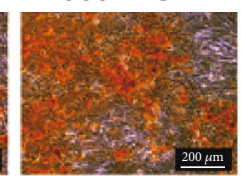

(a)

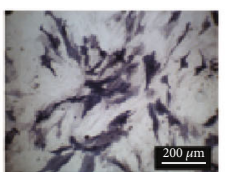

(b)

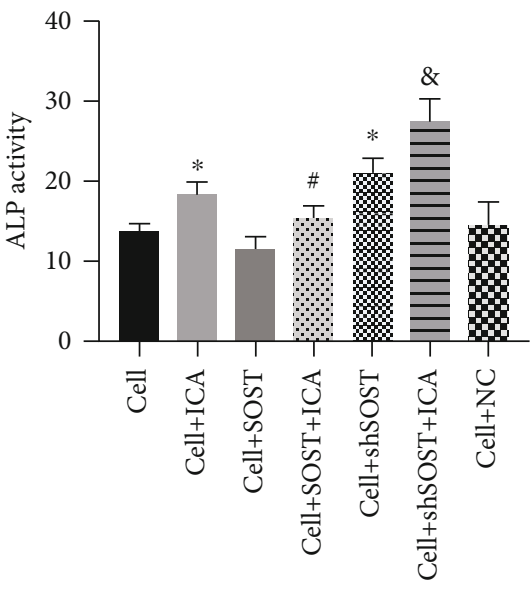

(c)

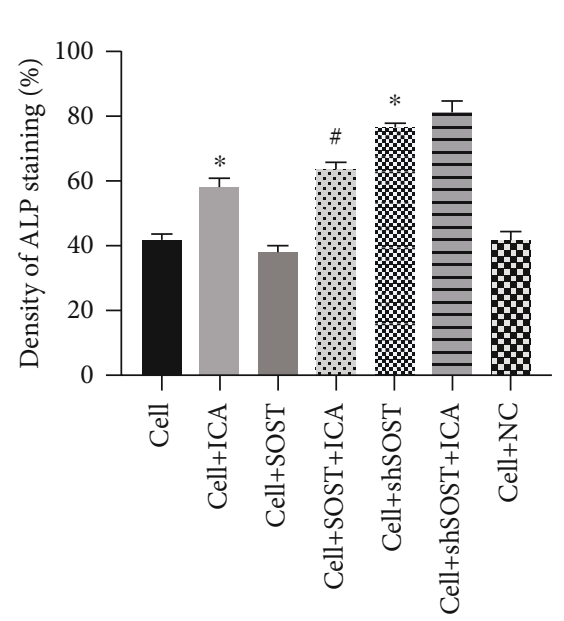

(d)

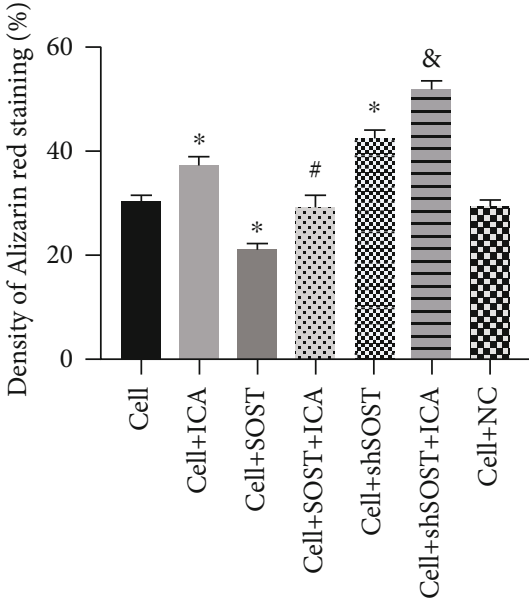

(e)

Figure 4: Effects of ICA and sclerostin on the osteogenic differentiation of BMSCs. (a) Alizarin red staining, (b) alkaline phosphatase staining, (c) ALP activity in the indicated groups, and (d, e) ratio of control in ALP staining and Alizarin red staining. All data are presented as mean \pm SEM. ${ }^{*} P<0.05$ compared to the control group, ${ }^{\#} P<0.05$ compared to the sclerostin overexpression group, and ${ }^{\&} P<0.05$ compared to the sclerostin knockdown group.

upregulated in the SOST-overexpressing and SOST-knockdown cells following ICA treatment (Figure 5). Taken together, sclerostin inhibits the osteogenic potential of BMSCs, which can be reversed by ICA.

3.4. ICA Enhances the Antioxidant Response in BMSCs. Oxidative stress is one of the underlying causes of OP and is known to inhibit osteogenesis [22]. To determine whether ICA modulates oxidative stress in the BMSCs, we analyzed the expression levels of antioxidant factors including Prdx1, Cata, and Nqo1 in the differentially treated cells. As shown in Figure 6, both ICA treatment and SOST knockdown significantly upregulated Prdx1, Cata, and Nqo1 mRNA levels on days 4 and 7 postosteogenic induction compared to the control group, whereas SOST overexpression had the opposite effect. Furthermore, ICA treatment augmented the antioxidant response in the SOST-knockdown BMSCs and restored the same in cells overexpressing SOST. Taken together, ICA promotes the osteogenic differentiation of BMSCs by mitigating oxidative stress.

3.5. ICA Promotes Osteogenesis via the Sclerostin/Wnt/ $\beta$ Catenin Signaling Pathway. To further elucidate the mecha- nistic basis of ICA action, we next analyzed the expression levels of key Wnt/ $\beta$-catenin pathway intermediates, such as $\beta$-catenin, GSK- $3 \beta$, and p-GSK-3 $\beta$. As shown in the immunoblots in Figure 7, both ICA and SOST-shRNA significantly upregulated $\beta$-catenin and $\mathrm{p}-\mathrm{GSK}-3 \beta$ proteins on days 4 and 7 of culture compared to the control group. In contrast, SOST overexpression downregulated these factors at the same time points. Consistent with the findings so far, ICA increased the expression of $\mathrm{Wnt} / \beta$-catenin pathway factors in BMSCs regardless of the SOST expression status. Taken together, ICA reverses the antiosteogenic activity of sclerostin by activating the $\mathrm{Wnt} / \beta$-catenin signaling pathway.

\section{Discussion}

The incidence of osteoporotic fractures is steadily increasing due to a globally aging population and is associated with reduced quality of life as well as significant socioeconomic burden [23]. Therefore, it is vital to explore novel treatment options for OP that are highly effective and have minimal side effects. We found that sclerostin inhibited BMSC proliferation and osteogenic differentiation, which was reversed by ICA treatment. ICA not only upregulated osteogenesis- 

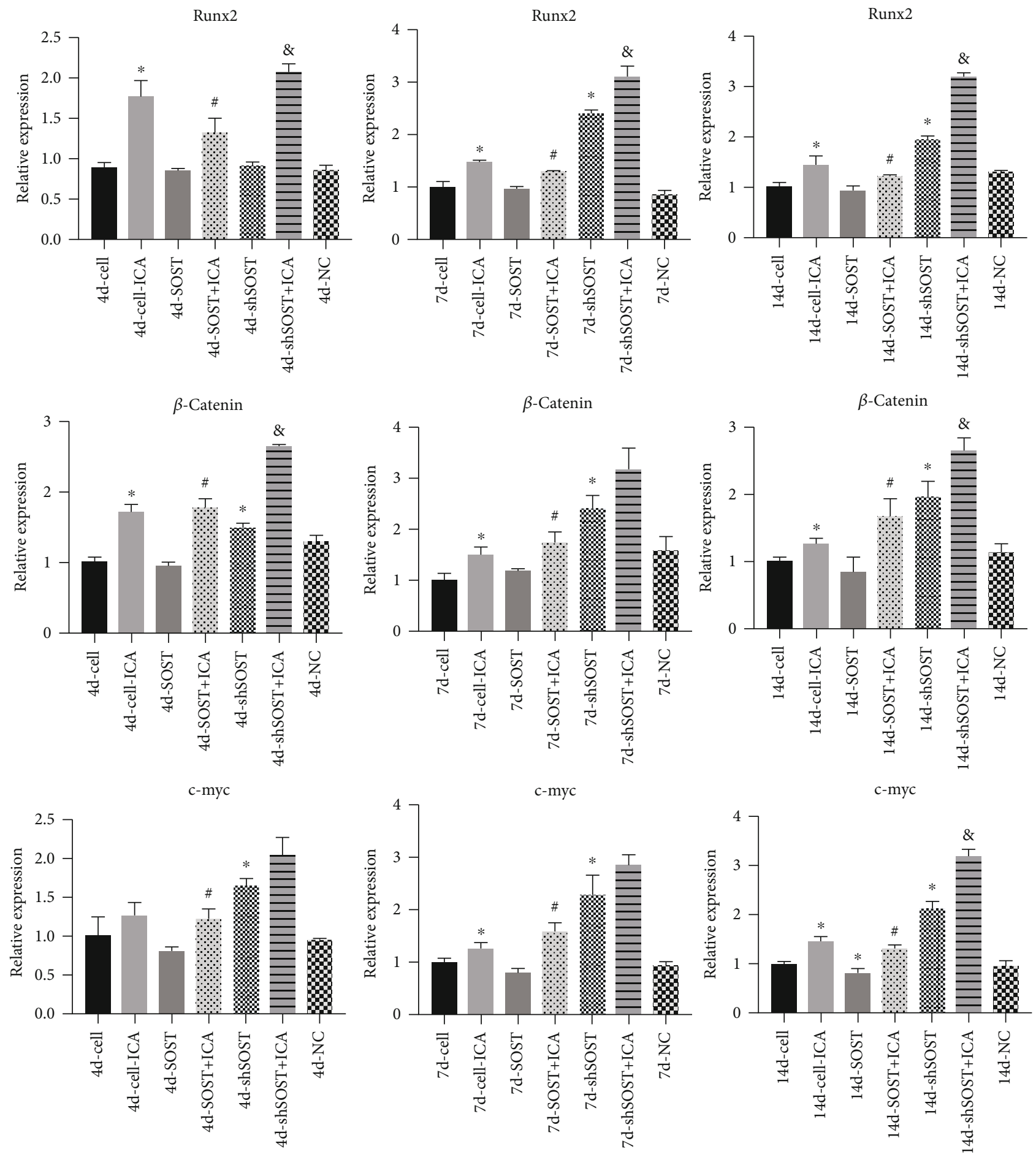

FIgURE 5: The expression of Runx2, $\beta$-catenin, and c-myc mRNAs at the different time points (4, 7, and 14 days) of osteogenic induction in the indicated groups. All data are presented as mean \pm SEM. ${ }^{*} P<0.05$ compared to the control group, ${ }^{\#} P<0.05$ compared to the sclerostin overexpression group, and ${ }^{8} P<0.05$ compared to the sclerostin knockdown group.

related genes including Runx2, $\beta$-catenin, and $\mathrm{c}$-myc but also increased the expression of antioxidant factors (Prdx1, Cata, and Nqo1). Furthermore, ICA activated the Wnt/ $\beta$-catenin pathway in SOST-overexpressing BMSCs. Thus, ICA may promote osteogenic differentiation of BMSCs by activating the sclerostin/Wnt $/ \beta$-catenin signaling pathway.
Sclerostin is secreted by bone cells and inhibits differentiation of osteoblasts [24]. SOST-/- mice display accelerated bone formation, as well as increased bone mass and strength [25]. Furthermore, blocking sclerostin with specific antibodies can restore bone mass during estrogen deficiency [26]. To the best of our knowledge, this is the 

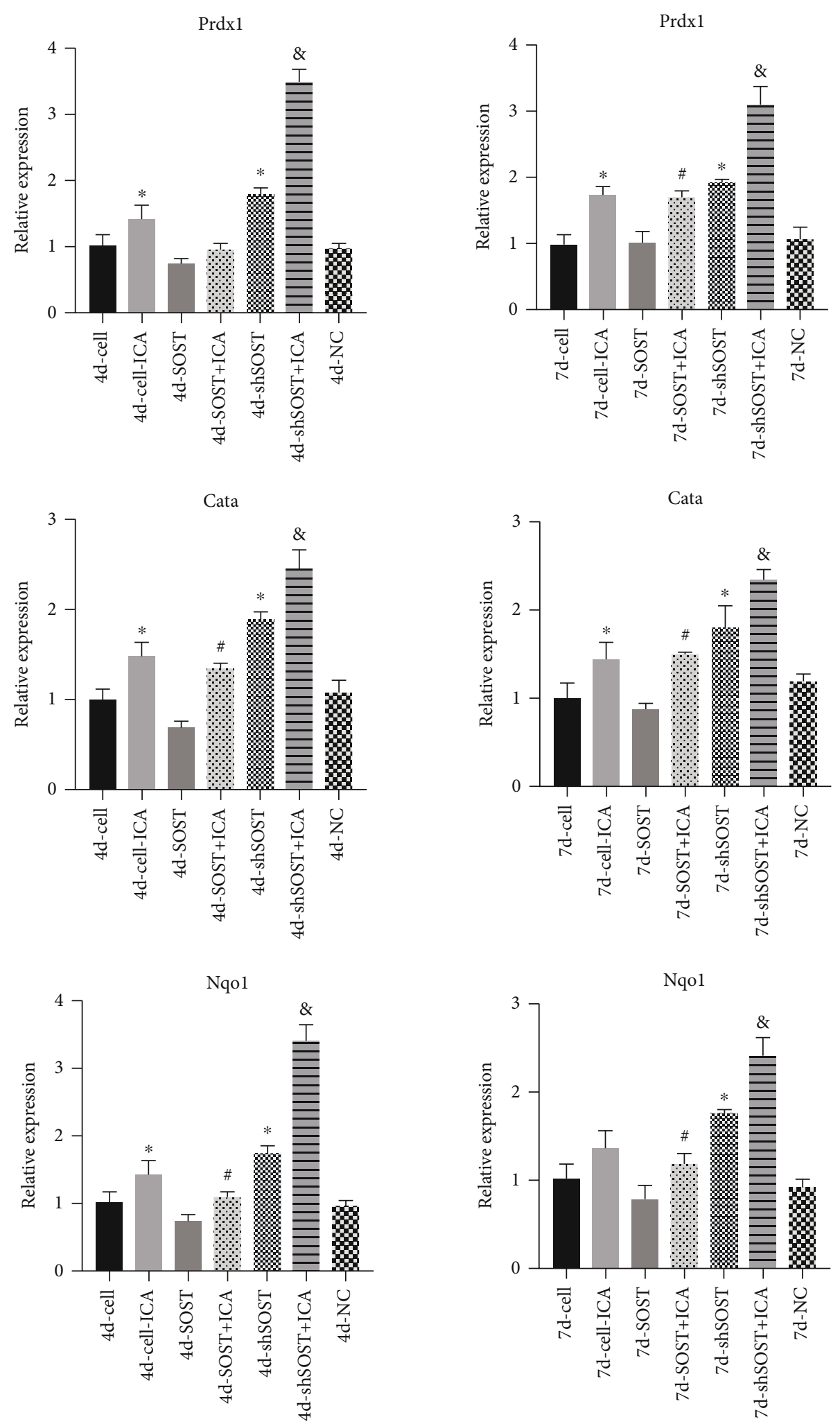

Figure 6: Prdx1, Cata, and Nqo1 mRNA levels in the BMSCs at the different time points (4 and 7 days) of osteogenesis in the indicated groups. All data are presented as mean \pm SEM. ${ }^{*} P<0.05$ compared to the control group, ${ }^{*} P<0.05$ compared to the sclerostin overexpression group, and ${ }^{\&} P<0.05$ compared to the sclerostin knockdown group.

first study to directly assess the role of sclerostin in the osteogenic differentiation of BMSCs in vitro. Sclerostin overexpression significantly inhibited the proliferation of
rBMSCs and downregulated osteogenic factors, which was neutralized by ICA, indicating a novel regulatory pathway of osteogenic differentiation. 

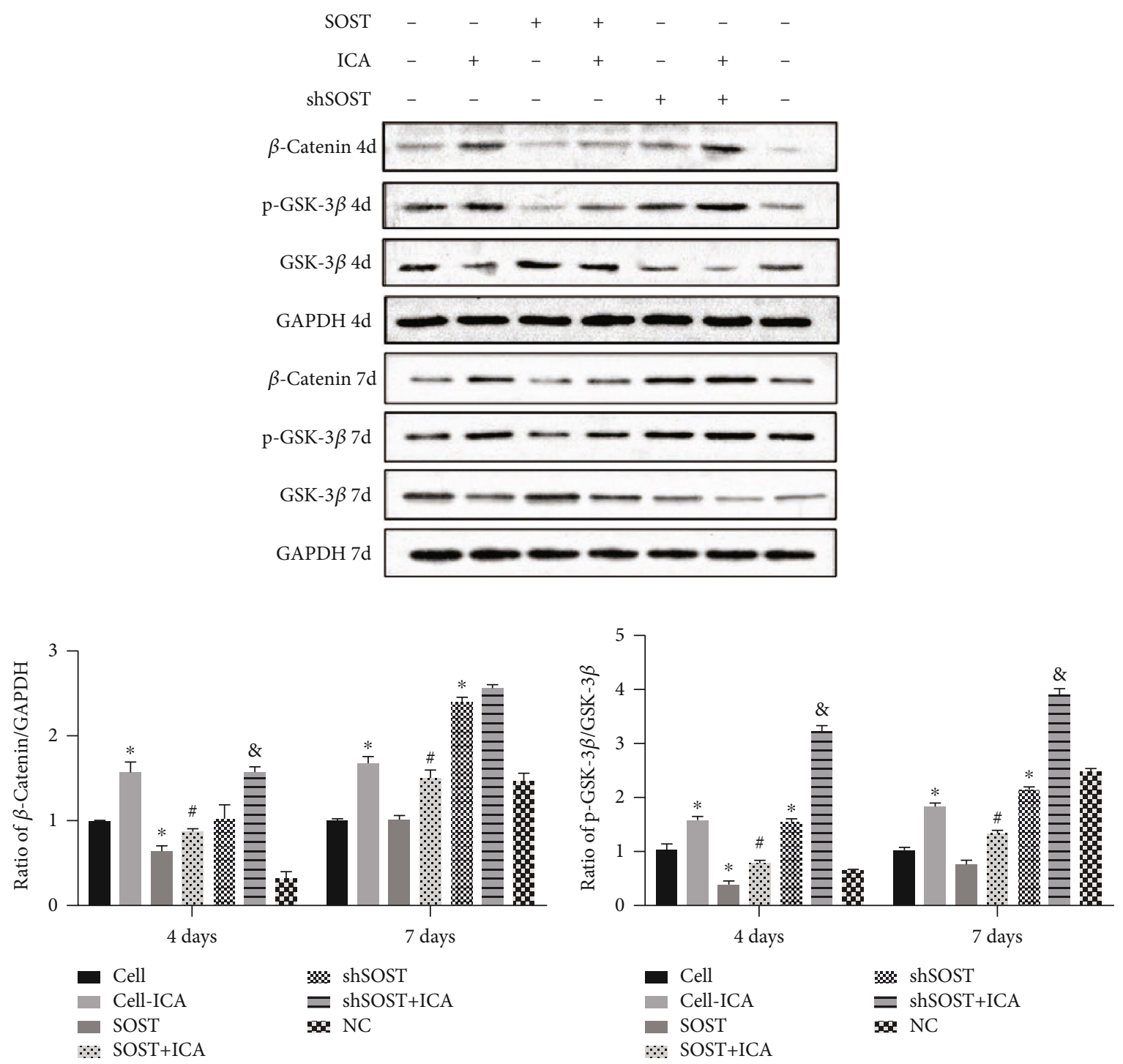

FIGURE 7: Immunoblot showing $\beta$-catenin, GSK-3 $\beta$, and p-GSK-3 $\beta$ protein levels in the BMSCs cultured for 4 and 7 days. All data are presented as mean \pm SEM. ${ }^{*} P<0.05$ compared to the control group, ${ }^{\#} P<0.05$ compared to the sclerostin overexpression group, and ${ }^{\&} P<0.05$ compared to the sclerostin knockdown group.

The transcription factor Runx 2 is necessary for osteoblast differentiation and bone formation and is an early indicator of the same [27]. Previous studies have shown that Runx2 induces differentiation and migration of osteoblasts and chondrocytes via the PI3K-Akt signaling pathway [28]. cmyc is a key downstream component of the $\mathrm{Wnt} / \beta$-catenin pathway and mediates cell proliferation. Indo et al. [29] reported that the inhibition of c-myc in mature osteoclasts reduces bone-resorbing activity and downregulates the neutral amino acid transporter (B0), which in turn suppresses osteoclastogenesis. We found that sclerostin significantly downregulated Runx 2 and c-myc, whereas ICA restored the expression of both. Thus, ICA promotes osteogenic differentiation of BMSCs by regulating the expression of sclerostin.

Oxidative stress plays an important role in osteoporosis [30] by accelerating apoptosis of BMSCs, osteoblasts, and osteoclasts as well as promoting the proliferation and differ- entiation of osteoclasts [31]. Prdx1, Cata, and Nqo1 are antioxidants [32] that clear ROS and suppress oxidative stress [33]. In the present study, sclerostin significantly downregulated the above factors and ICA had the opposite effect, indicating that inhibition of oxidative stress is one of the mechanisms through which ICA may promote osteogenic differentiation of BMSCs.

The Wnt/ $\beta$-catenin signaling pathway mediates BMSC proliferation and osteoblast differentiation. Following interaction of the Wnt glycoprotein with frizzled (FZD) and low-density lipoprotein receptor-related protein 5/6 (LRP5/6) [34], axin is recruited to the receptor complex and binds to the phosphorylation site of LRP. This leads to complex dissociation and phosphorylation of glycogen synthase kinase $3 \beta$ (p-GSK3 $\beta$ ), resulting in the cytosolic accumulation of $\beta$-catenin and nuclear translocation, eventually leading to the transcriptional activation of the target genes 


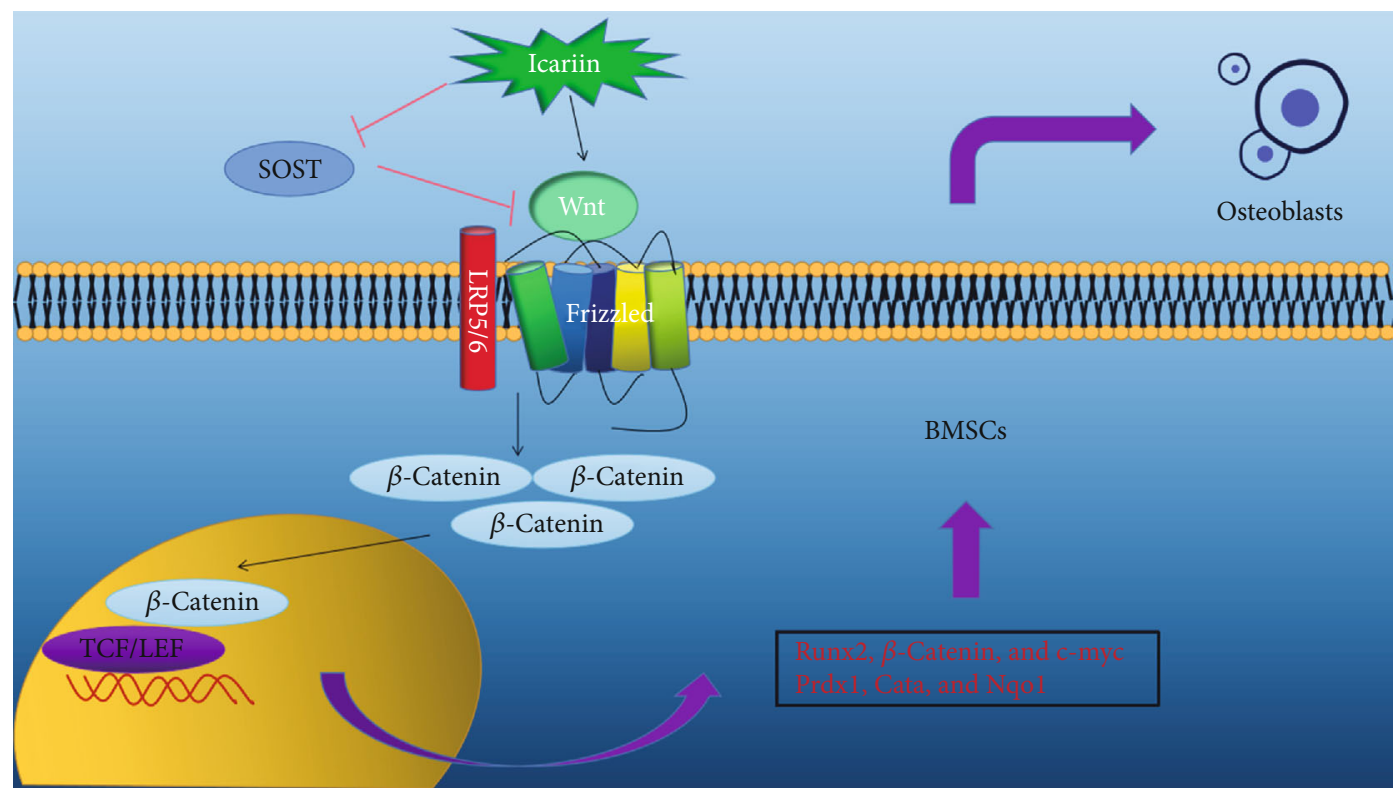

Figure 8: Schematic diagram.

[35]. Sclerostin downregulated the expression of $\beta$-catenin and $\mathrm{p}-\mathrm{GSK}-3 \beta$, which were increased by ICA treatment. Thus, ICA promotes osteogenesis via the sclerostin/Wnt/ $\beta$-catenin signaling pathway (schematic diagram shown in Figure 8).

\section{Conclusions}

In summary, ICA promotes osteogenic differentiation of BMSCs by inhibiting the action of sclerostin by activating the $\mathrm{Wnt} / \beta$-catenin pathway. It can potentially enhance the survival and differentiation of transplanted BMSCs in situ and improve the efficacy of BMSC-based regenerative therapy for OP.

\section{Data Availability}

The data used to support the results of this study can be obtained from the corresponding author according to the requirements.

\section{Conflicts of Interest}

All the authors declare they have no competing interests.

\section{Authors' Contributions}

Jianliang Gao and Shouyu Xiang contributed equally to this work.

\section{Acknowledgments}

This work was supported by the National Natural Science Foundation of China (No. 81574002) and Guangzhou Municipal Science and Technology Fund (No. 201704020032).

\section{References}

[1] K. E. Ensrud and C. J. Crandall, "Osteoporosis," Annals of Internal Medicine, vol. 167, no. 3, pp. ITC17-ITC32, 2017.

[2] I. Reid, "A broader strategy for osteoporosis interventions," Nature Reviews Endocrinology, vol. 16, no. 6, pp. 333-339, 2020.

[3] S. Abtahi, J. H. M. Driessen, P. Vestergaard et al., "Secular trends in major osteoporotic fractures among 50+ adults in Denmark between 1995 and 2010," Osteoporosis International, vol. 30, no. 11, pp. 2217-2223, 2019.

[4] N. B. Watts, J. T. Grbic, N. Binkley et al., "Invasive oral procedures and events in postmenopausal women with osteoporosis treated with denosumab for up to 10 years," The Journal of Clinical Endocrinology and Metabolism, vol. 104, no. 6, pp. 2443-2452, 2019.

[5] P. J. Voss, D. Steybe, P. Poxleitner et al., "Osteonecrosis of the jaw in patients transitioning from bisphosphonates to denosumab treatment for osteoporosis," Odontology, vol. 106, no. 4, pp. 469-480, 2018.

[6] A. Qadir, S. Liang, Z. Wu, Z. Chen, L. Hu, and A. Qian, "Senile osteoporosis: the involvement of differentiation and senescence of bone marrow stromal cells," International Journal of Molecular Sciences, vol. 21, no. 1, p. 349, 2020.

[7] Y. Liu, L. Ming, H. Luo et al., "Integration of a calcined bovine bone and BMSC-sheet 3D scaffold and the promotion of bone regeneration in large defects," Biomaterials, vol. 34 , no. 38, pp. 9998-10006, 2013.

[8] Z.-W. Luo, F.-X.-Z. Li, Y.-W. Liu et al., "Aptamer-functionalized exosomes from bone marrow stromal cells target bone to promote bone regeneration," Nanoscale, vol. 11, no. 43, pp. 20884-20892, 2019.

[9] J. Delgado-Calle, A. Y. Sato, and T. Bellido, "Role and mechanism of action of sclerostin in bone," Bone, vol. 96, pp. 29-37, 2017.

[10] K. Maeda, Y. Kobayashi, M. Koide et al., "The regulation of bone metabolism and disorders by Wnt signaling," 
International Journal of Molecular Sciences, vol. 20, no. 22, p. 5525, 2019.

[11] Z. C. Ryan, T. A. Craig, M. McGee-Lawrence, J. J. Westendorf, and R. Kumar, "Alterations in vitamin D metabolite, parathyroid hormone and fibroblast growth factor-23 concentrations in sclerostin-deficient mice permit the maintenance of a high bone mass," The Journal of Steroid Biochemistry and Molecular Biology, vol. 148, pp. 225-231, 2015.

[12] Y. Liu, X. Wang, H. Chang et al., "Mongolian medicine echinops prevented postmenopausal osteoporosis and induced ER/AKT/ERK pathway in BMSCs," Bioscience Trends, vol. 12, no. 3, pp. 275-281, 2018.

[13] H. Sheng, X.-f. Rui, C.-J. Sheng et al., "A novel semisynthetic molecule icaritin stimulates osteogenic differentiation and inhibits adipogenesis of mesenchymal stem cells," International Journal of Medical Sciences, vol. 10, no. 6, pp. 782-789, 2013.

[14] J. Jiang, J. Li, and X. Jia, "The antiosteoporotic activity of central-icaritin (CIT) on bone metabolism of ovariectomized rats," Molecules, vol. 19, no. 11, pp. 18690-18704, 2014.

[15] A. Yang, C. Yu, F. You, C. He, and Z. Li, "Mechanisms of Zuogui pill in treating osteoporosis: perspective from bone marrow mesenchymal stem cells," Evidence-Based Complementary and Alternative Medicine, vol. 2018, Article ID 3717391, 8 pages, 2018.

[16] H. Zhao, X. Li, D. Zhang et al., "Integrative Bone Metabolomics-Lipidomics Strategy for Pathological Mechanism of Postmenopausal Osteoporosis Mouse Model," Scientific Reports, vol. 8, no. 1, article 16456, 2018.

[17] X. Li, J. Zhan, Y. Hou et al., "Coenzyme Q10 Regulation of Apoptosis and Oxidative Stress in $\mathrm{H} 2 \mathrm{O} 2$ Induced BMSC Death by Modulating the Nrf-2/NQO-1 Signaling Pathway and Its Application in a Model of Spinal Cord Injury," Oxidative Medicine and Cellular Longevity, vol. 2019, Article ID 6493081, 15 pages, 2019.

[18] Q. Wei, B. Wang, H. Hu et al., "Icaritin promotes the osteogenesis of bone marrow mesenchymal stem cells via the regulation of sclerostin expression," International Journal of Molecular Medicine, vol. 45, no. 3, pp. 816-824, 2020.

[19] X.-J. Chen, Y.-S. Shen, M.-C. He et al., "Polydatin promotes the osteogenic differentiation of human bone mesenchymal stem cells by activating the BMP $2-W n t / \beta$-catenin signaling pathway," Biomedicine \& Pharmacotherapy, vol. 112, article 108746, 2019.

[20] Y. Cao, B. Wang, D. Wang et al., "Expression of Sclerostin in Osteoporotic Fracture Patients Is Associated with DNA Methylation in the CpG Island of the SOST Gene," International journal of genomics, vol. 2019, Article ID 7076513, 8 pages, 2019.

[21] A. Y. H. Ng, Z. Li, M. M. Jones et al., "Regulator of G protein signaling 12 enhances osteoclastogenesis by suppressing Nrf2-dependent antioxidant proteins to promote the generation of reactive oxygen species," eLife, vol. 8, 2019.

[22] Q. Zhou, L. Zhu, D. Zhang et al., "Oxidative stress-related biomarkers in postmenopausal osteoporosis: a systematic review and meta-analyses," Disease Markers, vol. 2016, Article ID 7067984, 12 pages, 2016.

[23] P. Alejandro and F. Constantinescu, "A review of osteoporosis in the older adult: an update," Rheumatic Diseases Clinics of North America, vol. 44, no. 3, pp. 437-451, 2018.
[24] X. Li, M. S. Ominsky, K. S. Warmington et al., "Sclerostin antibody treatment increases bone formation, bone mass, and bone strength in a rat model of postmenopausal osteoporosis," Journal of Bone and Mineral Research, vol. 24, no. 4, pp. 578$588,2009$.

[25] J. Jürimäe, V. Karvelyte, L. Remmel et al., "Sclerostin, preadipocyte factor- 1 and bone mineral values in eumenorrheic adolescent athletes with different training patterns," Journal of Bone and Mineral Metabolism, 2020.

[26] S. Thiele, A. Hannemann, M. Winzer et al., "Regulation of sclerostin in glucocorticoid-induced osteoporosis (GIO) in mice and humans," Endocrine Connections, vol. 8, no. 7, pp. 923-934, 2019.

[27] Q. Yin, J. Wang, Q. Fu, S. Gu, and Y. Rui, "CircRUNX2 through has-miR-203 regulates RUNX2 to prevent osteoporosis," Journal of Cellular and Molecular Medicine, vol. 22, no. 12, pp. 6112-6121, 2018.

[28] N. Cai, C. Li, and F. Wang, "Silencing of LncRNA-ANCR promotes the osteogenesis of osteoblast cells in postmenopausal osteoporosis via targeting EZH2 and RUNX2," Yonsei Medical Journal, vol. 60, no. 8, pp. 751-759, 2019.

[29] Y. Indo, S. Takeshita, K.-A. Ishii et al., "Metabolic regulation of osteoclast differentiation and function," Journal of Bone and Mineral Research, vol. 28, no. 11, pp. 2392-2399, 2013.

[30] C. Cervellati, G. Bonaccorsi, E. Cremonini et al., "Oxidative stress and bone resorption interplay as a possible trigger for postmenopausal osteoporosis," BioMed Research International, vol. 2014, Article ID 569563, 8 pages, 2014.

[31] K. H. Baek, K. W. Oh, W. Y. Lee et al., "Association of oxidative stress with postmenopausal osteoporosis and the effects of hydrogen peroxide on osteoclast formation in human bone marrow cell cultures," Calcified Tissue International, vol. 87, no. 3, pp. 226-235, 2010.

[32] Y. Kumar, T. Biswas, G. Thacker et al., "BMP signaling-driven osteogenesis is critically dependent on Prdx-1 expressionmediated maintenance of chondrocyte prehypetrophy," Free Radical Biology \& Medicine, vol. 118, pp. 1-12, 2018.

[33] X. Li, J. Zhan, Y. Hou et al., "Coenzyme Q10 suppresses oxidative stress and apoptosis via activating the Nrf-2/NQO-1 and $\mathrm{NF}-\kappa \mathrm{B}$ signaling pathway after spinal cord injury in rats," American Journal of Translational Research, vol. 11, no. 10, pp. 6544-6552, 2019.

[34] Y. Zhu, Y. Wang, Y. Jia, J. Xu, and Y. Chai, “Catalpol promotes the osteogenic differentiation of bone marrow mesenchymal stem cells via the Wnt/ $\beta$-catenin pathway," Stem Cell Research \& Therapy, vol. 10, no. 1, p. 37, 2019.

[35] L. D. Antika, E.-J. Lee, Y.-H. Kim et al., "Dietary phlorizin enhances osteoblastogenic bone formation through enhancing $\beta$-catenin activity via GSK-3 $\beta$ inhibition in a model of senile osteoporosis," The Journal of Nutritional Biochemistry, vol. 49, pp. 42-52, 2017. 